\title{
Hyperacute graft-vs.-host disease after related HLA-identical umbilical cord blood transplantation
}

Kuskonmaz B, Gocer S, Ersoy-Ewans S, Cetin-Ozman F, Cetin M, Uckan D. Hyperacute graft-vs.-host disease after related HLA-identical umbilical cord blood transplantation.

Pediatr Transplantation 2007: 11:818-820. @ 2007 Blackwell Munksgaard

Abstract: haGVHD has been described following bone marrow and peripheral blood stem cell transplantation and in a single case who received unrelated HLA mismatched $\mathrm{CB}$. An unusual case of haGVHD following HLA 6/6-matched sibling CBT in a child with AML is presented. The development of haGVHD in a fully matched CBT and without precipitating factors may suggest the role of still undefined and perhaps individual contributory factors.

\author{
Baris Kuskonmaz', Safak Gocer ${ }^{2}$, \\ Sibel Ersoy-Ewans ${ }^{3}$, Fatma Cetin \\ Ozman $^{3}$, Mualla Cetin ${ }^{1}$ and Duygu \\ Uckan ${ }^{4}$ \\ ${ }^{1}$ Pediatric Hematology Unit, Faculty of Medicine, \\ Hacettepe University, Ankara, Turkey, ${ }^{2}$ Pathology \\ Unit, Department of Pediatrics, Faculty of Medicine, \\ Hacettepe University, Ankara, Turkey, ${ }^{3}$ Department of \\ Dermatology, Faculty of Medicine, Hacettepe \\ University, Ankara, Turkey, ${ }^{4}$ Pediatric Hematology- \\ Bone Marrow Transplantation Unit, Hacettepe \\ University Faculty of Medicine, Ihsan Dogramaci \\ Childrens Hospital, Ankara, Turkey \\ Key words: graft-vs.-host disease - hyperacute - \\ cord blood transplantation \\ Duygu Uckan, Pediatric Bone Marrow Transplantation \\ Unit, Hacettepe University, Ankara, Turkey \\ Tel.: +90 3123051979 \\ Fax: +90 3123243284 \\ E-mail: duckan@hacettepe.edu.tr \\ Accepted for publication 26 June 2007
}

The term "hyperacute" refers to the pre-engraftment manifestation of GVHD (1). The main clinical manifestations of haGVHD are unexplained fever, skin rash, hepatic dysfunction, and diarrhea (1). Sullivan et al. (2) first described the clinical manifestations of haGVHD in allogeneic BMT in 1986. To the best of our knowledge, the present case is the first to have developed haGVHD following HLA-matched sibling CBT.

\footnotetext{
Abbreviations: AML, acute myeloid leukemia; BMT, bone marrow transplantation; $\mathrm{CB}$, cord blood; $\mathrm{CBT}$, cord blood transplantation; CsA, cyclosporine A; GVHD, graftvs.-host disease; haGVHD, hyperacute GVHD; HSC, hematopoietic stem cells; HSCT, hematopoietic stem cell transplantation; MP, methylprednisolone; SOS, sinusoidal obstruction syndrome; VOD, veno-occlusive disease.
}

\section{Case report}

A four-yr-old girl with AML in second complete remission underwent CBT from her HLA-identical brother in November 2005. The conditioning regimen consisted of i.v. busulphan (busulfex, $12.8 \mathrm{mg} / \mathrm{kg}$ over four days), melphalan $(140 \mathrm{mg}$ / $\mathrm{m}^{2} \times 1$ day), and cyclophosphamide $(120 \mathrm{mg} /$ $\mathrm{kg}$ ). GVHD prophylaxis consisted of CsA, beginning with an i.v. dose of $3 \mathrm{mg} / \mathrm{kg} /$ day on day -2 ; with dose then adjusted according to blood levels. The CB nucleated cell dose was $12.2 \times 10^{7}$ cells $/ \mathrm{kg}$ and $6 \times 10^{5} \mathrm{CD} 34$ cells $/ \mathrm{kg}$ was given. The patient developed fever on day +3 for which she was treated with i.v. antibiotics. The fever persisted and she developed widespread erythematous plaques on her neck, face, axilla, and waist. Purplish-red lichenoid papules were then noted on the trunk, glutea, and extremities on day +6 (Fig. 1). Histopathological examination of lesional skin punch 


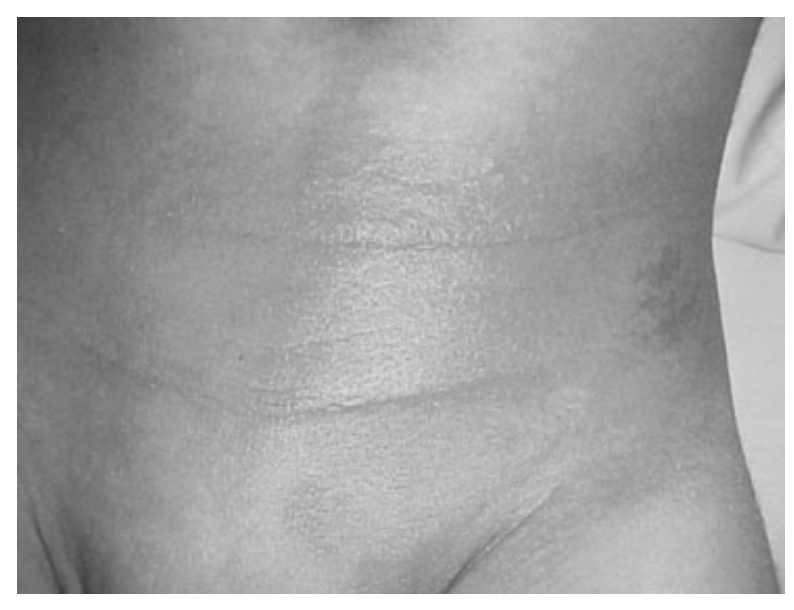

Fig. 1. Skin findings of the patient.

biopsy revealed focal vacuolization of the basal cell layer, spongiosis, and dyskeratotic keratinocytes in epidermis. Dyskeratosis was present in hair follicles and occasional perivascular lymphocytes were noted. The eccrine glands and vascular structures were normal. A histopathological diagnosis of acute GVHD, grade II was made (Fig. 2). MP was initially started at a dose of $2 \mathrm{mg} / \mathrm{kg}$ and was increased to $5 \mathrm{mg} / \mathrm{kg}$ upon the appearance of watery diarrhea. Fever disappeared, skin lesions regressed following two days of MP treatment, and diarrhea resolved completely in five days. Physical examination, urine analysis, chest X-ray, and throat and recurrent blood cultures were negative, and no pathogenic microorganisms were isolated from stool specimens. Weekly assessment for galactomannan antigenemia and cytomegalovirus viremia failed to show an infectious etiology. MP dose was

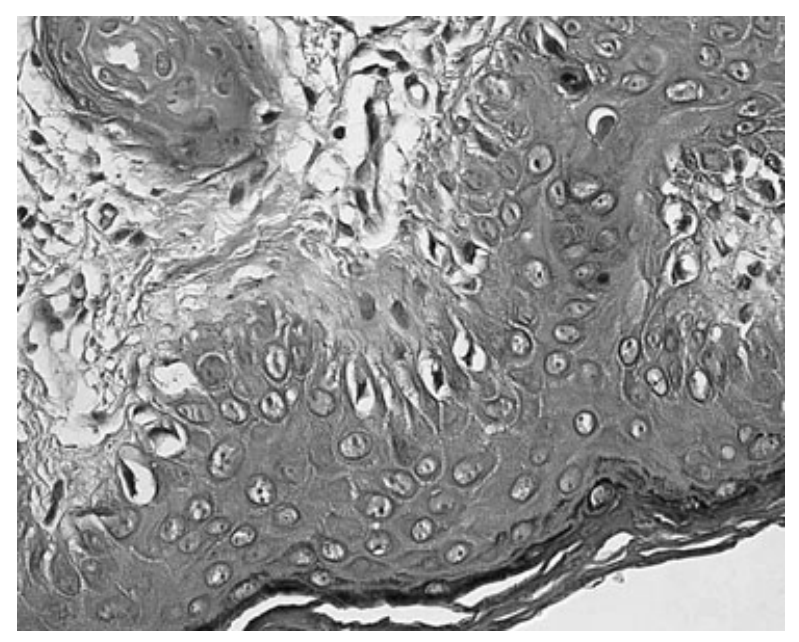

Fig. 2. Vacuolization of basal layer, spongiosis, and dyskeratotic keratinocytes in epidermis of tangential section of skin biopsy. decreased to $1 \mathrm{mg} / \mathrm{kg}$ on day +17 and was discontinued on day +34 . Neutrophil engraftment occurred on day +17 , platelets +26 , and the last red blood cell transfusion was administered on day +15 . Full donor hematopoietic chimerism was determined by molecular method and cytogenetics on day +26 . At the sevenmonth follow-up, the patient was in full remission with $100 \%$ donor chimerism, and off immunosuppressive treatment.

\section{Discussion}

Cord blood is an alternative source of HSC, and has some advantages over bone marrow and peripheral blood cells. The "naive" nature of $\mathrm{CB}$ lymphocytes permits the use of HLA-mismatched grafts at 1-2 loci without higher risk for severe GVHD relative to BMT from a fullmatched unrelated donor (3). Investigators have reported decreased incidences of acute and chronic GVHD following CBT (4). haGVHD has not been described following CBT except in one case of mismatched CBT associated with Ralstonia pickettii bacteremia (5).

The overall incidence of haGVHD ranges between $14 \%$ and $38 \%$ in allogenic settings $(1,6)$. The diagnostic criteria of haGVHD include: (i) unexplained fever, before engraftment, of $>38.3^{\circ} \mathrm{C}$ on two occasions (each occasion lasting more than three days) with a lack of resolution after a minimum of three days of treatment with antibiotics and antifungal agents, including amphotericin B; (ii) rapid development of skin rash, before engraftment, with the exclusion of other causes, such as drug eruption; (iii) hepatic dysfunction before engraftment, especially increased alkaline phosphatase and bilirubin levels, with the exclusion of other causes, such as VOD/SOS, drug-induced hepatitis, or right-sided heart failure; and (iv) development of mucoid, greenish diarrhea, with more than five stools/day, with the exclusion of mucositis (1). The diagnosis is established based on the presence of the above-mentioned criterion (i) in addition to one of criteria (ii)-(iv) with or without histological confirmation, and is supported by the resolution of the above clinical findings with steroid treatment (1). The diagnosis of haGVHD in the present case was made due to the presence of fever without focus of infection, appearance of skin rash before engraftment, diarrhea, and improvement of symptoms following steroid treatment. In addition to these findings, histological confirmation was made by skin biopsy (Fig. 2). In this patient, the skin rash appeared on day 6 as has been reported in 
previous studies (day 4-7) (1). Alternatively, the clinical findings in this patient were also suggestive of engraftment syndrome/peri-engraftment clinical abnormalities (7). However, the neutrophil and platelet engraftment days were +17 and +26 , respectively, the clinical findings of the patient occurred in the first post-transplant week, and skin biopsy was consistent with acute GVHD. Therefore, engraftment syndrome was not considered in this patient. Moreover, the diagnosis of haGVHD in the present patient is also in accordance with the definition of haGVHD in a very recent report by Saliba et al. (8). The authors have defined haGVHD as a diagnosis of acute GVHD within the first 14 days after HSCT (8).

The risk factors for haGVHD include: a mismatched related or matched unrelated donor, a myeloablative conditioning regimen, more than five prior chemotherapy regimens, donor-recipient sex mismatch (8), use of an alternative donor (1), and sudden discontinuation of post-transplant immunosuppression (2). Donor pretreatment with granulocyte colony-stimulating factor has been shown to suppress haGVHD in murine models and clinical studies $(9,10)$.

The results of CBT have been found to be comparable to those obtained by BMT (11), whereas time to engraftment is delayed and the incidence and severity of GVHD are reduced (4, 12, 13). The apparent reduced immunogenicity of the $\mathrm{CB}$ was evidenced by the lower than expected incidence of severe GVHD reported following both related and unrelated CBT, even in mismatched transplants $(14,15)$. The present case of haGVHD was unusual considering the use of HLA-identical CB graft from a sibling donor, standard immunosuppressive treatment, myeloablative conditioning, and the disease remission status. To the best of our knowledge, the present case is the first to have developed haGVHD after related and matched CBT.

The use of high-dose corticosteroids (i.v. $3-5 \mathrm{mg} / \mathrm{kg} /$ day) for five days was found to be very effective in treating haGVHD, leading to abrupt resolution of fever in the majority of patients (1). The clinical response to steroid therapy was remarkable in the present case, as expected.

It has been shown that haGVHD is associated with later development of acute and chronic GVHD, lower response rate to first-line therapy and a higher rate of non-relapse mortality in patients with a mismatched related or matched unrelated donor graft $(1,7,8)$. The lack of acute and chronic GVHD in the present case was attributed to the slow tapering of steroids and the adjustment of CsA dose to blood levels. At the seventh-month follow-up, the patient was in full remission with $100 \%$ donor chimerism, and off immunosuppressive treatment.

In conclusion, the unexpected development of haGVHD following HLA-matched CBT from a related donor is described in this report. The development of haGVHD in a fully matched CBT and without precipitating factors may suggest the role of still undefined and perhaps individual contributory factors.

\section{References}

1. Kim DH, Sohn SK, Kim JG, Suh JS, Lee KS, Lee KB. Clinical impact of hyperacute graft-versus-host disease on results of allogeneic stem cell transplantation. Bone Marrow Transplant 2004: 33: 1025-1030.

2. Sullivan KM, Deeg HJ, Sanders J, et al. Hyperacute graft-vhost disease in patients not given immunosuppression after allogeneic marrow transplantation. Blood 1986: 67: 1172-1175.

3. Cohen Y, Nagler A. Umbilical cord blood transplantation how, when and for whom? Blood Rev 2004: 18: 167-179.

4. Rocha V, Wagner JE Jr., Sobocinski KA, et al. Comparison of graft-versus-host disease in children transplanted with HLA identical sibling umblical cord blood versus HLA identical sibling bone marrow transplant. N Engl J Med 2000: 342: 1846-1854.

5. Woo PC, Wong SS, Yuen KY. Ralstonia pickettii bacteraemia in a cord blood transplant recipient. New Microbiol 2002: 25 : 97-102.

6. Huang X, Chen Y, Guo N, et al. Hyperacute graft versus host disease after allo-stem cell transplantation, analysis of 118 cases. Zhonghua Yi Xue Za Zhi 2002: 82: 511-514.

7. Choi SJ, Lee KH, Lee JH, et al. Peri-engraftment clinical abnormalities following allogeneic hematopoietic cell transplantation: A retrospective review of 216 patients. Bone Marrow Transplant 2003: 32: 809-813.

8. Saliba RM, De Lima M, Giralt S, et al. Hyperacute GVHD: Risk factors, outcomes, and clinical implications. Blood 2007: 109: 2751-2758.

9. Pan L, Teshima T, Hill GR, et al. Hyperacute graft-versushost disease and NKT cells. Blood 1999: 93: 4071-4078.

10. Reddy V, Hill GR, PAN L, et al. G-CSF modulates cytokine profile of dendritic cells and decreases acute graft-versus-host disease through effects on the donor rather than the recipient. Transplantation 2000: 69: 691-693.

11. Gluckman E, Rocha V, Arcese W, et al. Eurocord Group. Factors associated with outcomes of unrelated cord blood transplant: Guidelines for donor choice. Exp Hematol 2004: 32: 397-407.

12. Gluckman E, Rocha V, Boyer-chammard A, et al. Outcome of cord-blood transplantation from related and unrelated donors. Eurocord Transplant Group and the European Blood and Marrow Transplantation Group. N Engl J Med 1997: 337: 373-381.

13. Kurtzber J, Laughlin M, Graham ML, et al. Placental blood as a source of hematopoietic stem cells for transplantation into unrelated recipients. N Engl J Med 1996: 335: 157-166.

14. Han P, Hodge G, Story C, XU X. Phenotypic analysis of functional T-lymphocyte subtypes and natural killer cells in human cord blood: Relevance to umbilical cord blood transplantation. Br J Haematol 1995: 89: 733-740.

15. Risdon G, Gaddy J, Horie M, Broxmeyer HE. Alloantigen priming induces a state of unresponsiveness in human umbilical cord blood T cells. Proc Natl Acad Sci USA 1995: 92: 24132417. 